Article

\title{
Elucidation of Molecular Mechanism of a Selective PPAR $\alpha$ Modulator, Pemafibrate, through Combinational Approaches of X-ray Crystallography, Thermodynamic Analysis, and First-Principle Calculations
}

\author{
Mayu Kawasaki ${ }^{1, \dagger}$, Akira Kambe ${ }^{1, \dagger}$, Yuta Yamamoto ${ }^{2}$, Sundaram Arulmozhiraja ${ }^{2}$, Sohei Ito ${ }^{1,3}$, \\ Yoshimi Nakagawa ${ }^{4,5}\left(\mathbb{D}\right.$, Hiroaki Tokiwa ${ }^{2,3,6} \oplus$, Shogo Nakano $\left.{ }^{1,3, *} \mathbb{(}\right)$ and \\ Hitoshi Shimano $3,4,5,7, *$ (D) \\ 1 Graduate Division of Nutritional and Environmental Sciences, University of Shizuoka, 52-1 Yada, Suruga-ku, \\ Shizuoka 422-8526, Japan; s18217@u-shizuoka-ken.ac.jp (M.K.); f16007@u-shizuoka-ken.ac.jp (A.K.); \\ itosohei@u-shizuoka-ken.ac.jp (S.I.) \\ 2 Department of Chemistry, Rikkyo University, 3-34-1 Nishi-Ikebukuro, Toshima-ku, Tokyo 171-8501, Japan; \\ yuta.abinitio.yamamoto@gmail.com (Y.Y.); raja@rikkyo.ac.jp (S.A.); tokiwa@rikkyo.ac.jp (H.T.) \\ 3 Japan Agency for Medical Research and Development-Core Research for Evolutional Science and \\ Technology (AMED-CREST), Chiyoda-ku, Tokyo 100-1004, Japan \\ 4 Department of Internal Medicine (Endocrinology and Metabolism), Faculty of Medicine, \\ University of Tsukuba, 1-1-1 Tennodai, Tsukuba, Ibaraki 305-8575, Japan; yosshy@md.tsukuba.ac.jp \\ 5 International Institute for Integrative Sleep Medicine (WPI-IIIS), University of Tsukuba, 1-1-1 Tennodai, \\ Tsukuba, Ibaraki 305-8575, Japan \\ 6 Research Center for Smart Molecules, Rikkyo University, 3-34-1 Nishi-Ikebukuro, Toshima-ku, \\ Tokyo 171-8501, Japan \\ 7 Life Science Center for Survival Dynamics, Tsukuba Advanced Research Alliance (TARA), \\ University of Tsukuba, 1-1-1 Tennodai, Tsukuba, Ibaraki 305-8577, Japan \\ * Correspondence: snakano@u-shizuoka-ken.ac.jp (S.N.); hshimano@md.tsukuba.ac.jp (H.S.); \\ Tel.: +81-54-264-5578 (S.N.); +81-29-863-2081 (H.S.) \\ + These authors contributed equally to this work.
}

Received: 28 November 2019; Accepted: 27 December 2019; Published: 6 January 2020

check for updates

\begin{abstract}
The selective PPAR $\alpha$ modulator (SPPARM $\alpha$ ) is expected to medicate dyslipidemia with minimizing adverse effects. Recently, pemafibrate was screened from the ligand library as an SPPARM $\alpha$ bearing strong potency. Several clinical pieces of evidence have proved the usefulness of pemafibrate as a medication; however, how pemafibrate works as a SPPARM $\alpha$ at the molecular level is not fully known. In this study, we investigate the molecular mechanism behind its novel SPPARM $\alpha$ character through a combination of approaches of X-ray crystallography, isothermal titration calorimetry (ITC), and fragment molecular orbital (FMO) analysis. ITC measurements have indicated that pemafibrate binds more strongly to PPAR $\alpha$ than to PPAR $\gamma$. The crystal structure of PPAR $\alpha$-ligand binding domain (LBD)/pemafibrate/steroid receptor coactivator-1 peptide (SRC1) determined at $3.2 \AA$ resolution indicates that pemafibrate binds to the ligand binding pocket (LBP) of PPAR $\alpha$ in a Y-shaped form. The structure also reveals that the conformation of the phenoxyalkyl group in pemafibrate is flexible in the absence of SRC1 coactivator peptide bound to PPAR $\alpha$; this gives a freedom for the phenoxyalkyl group to adopt structural changes induced by the binding of coactivators. FMO calculations have indicated that the accumulation of hydrophobic interactions provided by the residues at the LBP improve the interaction between pemafibrate and PPAR $\alpha$ compared with the interaction between fenofibrate and PPAR $\alpha$.
\end{abstract}


Keywords: pemafibrate; SPPARM $\alpha$; X-ray crystal structure; isothermal titration calorimetry; fragment molecular orbital theory

\section{Introduction}

Peroxisome proliferator-activated receptors (PPARs) are members of families of nuclear receptors, which play an important role in lipid metabolism. Three PPAR subtypes (PPAR $\alpha, \operatorname{PPAR} \gamma, \operatorname{PPAR} \delta)$ are expressed in different tissues. PPAR $\alpha$ is mainly expressed in metabolically active tissues, such as liver, kidney, heart, and muscle [1]. PPAR $\gamma$ is confirmed in white and brown adipocytes, and PPAR $\delta$ is expressed in many tissues ubiquitously with lower expression levels compared with the other two subtypes [1]. In this study, PPAR $\alpha$ and PPAR $\gamma$ were used as target molecules. For physiological functions, PPAR $\alpha$ regulates gene expression involved in peroxisomal and mitochondrial $\beta$-oxidation [2], whereas PPAR $\gamma$ modulates the expression involved in energy storage and utilization [1].

Because of these backgrounds, the activation of PPARs would be appropriate to medicate various diseases, such as dyslipidemia and type 2 diabetes. Several PPAR $\alpha$ and PPAR $\gamma$ agonists have been developed for now [1]. X-ray structural analysis would be helpful to figure out how the agonists activate PPARs at the molecular level. Many research groups succeeded in determining the cocrystal structures of PPARs with their agonists [3-9], suggesting that there is a common mechanism for the activation; AF2 interface is stabilized by binding of agonists to ligand-binding pocket (LBP) of PPARs ligand binding domain (LBD) [8], and this stabilization makes easy for PPARs to recruit coactivators. Although the activation mechanism appeared to be common among PPARs, LBP configuration is different among PPAR subtypes [10]. Thus, the binding affinity of PPAR agonists toward each PPAR subtypes can be regulated by changing their chemical structure [8]. Among the agonists, selective PPAR modulators that can activate PPAR $\alpha$ (SPPARM $\alpha$ ) [11] and PPAR $\gamma(\operatorname{SPPARM} \gamma)$ [12-15] selectively are expected to be alternatives of conventional PPAR agonists because they can reduce side effects. Currently, thiazolidinone [16-18] and fibrate [19-22] compounds have been developed to activate PPAR $\gamma$ and PPAR $\alpha$, respectively; in this study, we focused on fibrates.

In the fibrate class, fenofibric acid (fenofibrate) was developed as a PPAR $\alpha$ agonist for the treatment of dyslipidemia [23]. In clinical trials, the administration of fibrates could reduce the concentration of triglycerides (TG) and increase the concentration of high-density lipoprotein (HDL) in plasma [24,25]. However, fibrates such as fenofibrate and bezafibrate could not come up with a satisfactory outcome in preventing cardiovascular events in the limited group of dyslipidemic patients presumably due to weak potency and some adverse effects, such as myopathy and renal dysfunction [26]. The development of SPPARM $\alpha$ bearing high potency has been expected to overcome these disadvantages.

Recently, pemafibrate was identified as a SPPARM $\alpha$ and is shown to have strong potency. Pemafibrate was designed through the transactivation analysis of many substituents to improve its potency and selectivity toward PPAR $\alpha$ [27]. The activity was confirmed by in vitro and in vivo assay analysis-in cell-based assays, pemafibrate is shown to have over 2500-fold higher potency than that of the fenofibric acid [28]. Gene expression analysis also supported that treatment of pemafibrate activates PPAR $\alpha$ [29]. Preclinical study showed that pemafibrate markedly decreases the TG concentration and increases HDL-C levels compared with fenofibric acid [30]. In addition, pemafibrate exhibits no serious adverse effects, such as renal and hepatic disorders [28,31]. Combination therapy of pemafibrate and statin reduces TG levels significantly compared with statin monotherapy by reducing adverse effects [32]. These characteristic activities appear to be brought by unique Y-shaped structures of pemafibrate which consists of carboxylic, phenoxyalkyl, and 2-aminobenzoxasole groups [27]. By using in silico approaches, we suggested earlier that the Y-shaped pemafibrate interacts with PPAR $\alpha$ by fully occupying its LBP [33]. However, further studies are necessary to confirm the molecular mechanism of pemafibrate interaction with PPAR $\alpha$ due to the lack of cocrystal structure of PPAR $\alpha$ with pemafibrate. 
In this study, we elucidate the molecular mechanism behind SPPARM $\alpha$ nature of pemafibrate by a combination of approaches of isothermal titration calorimetry (ITC), X-ray crystallography, and fragment molecular orbital (FMO) method. ITC measurements indicated that pemafibrate is selective against PPAR $\alpha$. The crystal structure of the PPAR $\alpha$-LBD/pemafibrate/SRC1 complex revealed a unique binding mode of pemafibrate. Interaction energy analysis by FMO calculation between PPAR $\alpha$ and pemafibrate showed that pemafibrate formed many hydrophobic interactions with the residues sitting in the LBP site. Taken together, we confirmed the unique functionalities of pemafibrate based on structural and functional analysis approaches.

\section{Results}

\subsection{Estimation of Binding Affinity between PPARs-LBD and Two Fibrates}

At first, we attempted to indicate that pemafibrate selectively binds to PPAR $\alpha$ by in vitro assay. Here, ITC measurement is suitable to estimate the binding affinities. With this approach, dissociation constant $\left(K_{\mathrm{d}}\right)$ can be directly determined by monitoring heat generated by binding ligands to the target protein. The ITC measurement also provides the difference in enthalpy $(\Delta H)$ and entropy $(-T \Delta S)$ values due to complexation, which is helpful in understanding the nature of interactions.

In this study, we measured the binding affinities of two fibrates (pemafibrate and fenofibric acid) to PPARs-LBD (PPAR $\alpha$ - and PPAR $\gamma$-LBD) using ITC (Figure 1); as one of the fundamental and important members of the fibrates family, fenofibrate was used to compare the activity difference with pemafibrate broadly $[11,30,32,34]$. Here, PPAR $\gamma$-LBD was selected for a comparison of PPAR $\alpha$-LBD because fenofibrate activates PPAR $\alpha$ and PPAR $\gamma$, but not PPAR $\delta$ [11]. The thermodynamic parameters are shown in Table 1. The binding isotherms depicted in Figure 1 (sigmoidal curve1), suggest that the fibrates bind to the PPARs-LBD except fenofibric acid to PPAR $\gamma$-LBD. The interaction between fenofibric acid and PPAR $\gamma$-LBD was too weak to determine the thermodynamic parameters (Table 1). The obtained parameters indicate that pemafibrate selectively binds to PPAR $\alpha$-LBD as expected. In fact, the $K_{d}$ value obtained for the pemafibrate/PPAR $\alpha$-LBD complex is $0.13 \mu \mathrm{M}$ which is around 50and 70-fold lower than the values obtained for fenofibric acid/PPAR $\alpha$-LBD (7.37 $\mu \mathrm{M}$, Table 1), and pemafibrate/PPAR $\gamma$-LBD $(9.58 \mu \mathrm{M}$, Table 1), respectively.

Table 1. Thermodynamic parameters for the interaction between PPARs-LBD (PPAR $\alpha$-LBD and PPAR $\gamma$-LBD) and two fibrates (pemafibrate and fenofibric acid) at $25^{\circ} \mathrm{C}$ measured by ITC ${ }^{\text {a }}$.

\begin{tabular}{|c|c|c|c|c|c|}
\hline & $n$ & $K_{\mathrm{d}}$ & $\Delta G$ & $\Delta H$ & $-T \Delta S$ \\
\hline & & $\mu \mathrm{M}$ & & $\mathrm{kcal} / \mathrm{mol}$ & \\
\hline \multicolumn{6}{|c|}{ Pemafibrate } \\
\hline PPAR $\alpha$-LBD & $0.61 \pm 0.03$ & $0.13 \pm 0.04$ & $-9.37 \pm 0.02$ & $-12.3 \pm 0.6$ & $3.13 \pm 0.69$ \\
\hline $\operatorname{PPAR} \gamma$-LBD & $0.65 \pm 0.05$ & $9.58 \pm 1.85$ & $-6.83 \pm 0.41$ & $-17.1 \pm 1.0$ & $10.3 \pm 1.2$ \\
\hline \multicolumn{6}{|c|}{ Fenofibric Acid } \\
\hline $\operatorname{PPAR} \alpha-\mathrm{LBD}$ & $0.27 \pm 0.04$ & $7.37 \pm 2.68$ & $-7.02 \pm 0.25$ & $-23.2 \pm 2.3$ & $16.1 \pm 2.5$ \\
\hline PPAR $\gamma$-LBD & n.d. ${ }^{b}$ & n.d. & n.d. & n.d. & n.d. \\
\hline
\end{tabular}

Here, one question may emerge on how pemafibrate can bind to PPAR $\alpha$ selectively. To answer this question, we compared two energetic terms, $\Delta H$ and $-T \Delta S . \Delta H$ represents highly specific interactions, such as hydrogen bond and Van der Waals interactions [35]. $-T \Delta S$ indicates low specific interactions, including hydrophobic interaction [36]. The binding affinity of drug molecules could be improved by optimizing both $\Delta H$ and $-T \Delta S$ values; in fact, strong statins, such as rosuvastatin, have more favorable $\Delta H$ and $-T \Delta S$ values than classic statins [35]. The $\Delta G$ value of PPAR $\alpha$-LBD and pemafibrate, which is a summation of the $\Delta H$ and $-T \Delta S$ values, was lower than the $\Delta G$ value of PPAR $\alpha$-LBD and fenofibrate, and PPAR $\gamma$-LBD and pemafibrate (Table 1). This favorable $\Delta G$ value of PPAR $\alpha$-LBD and pemafibrate to form strong interactions appears to be brought by the optimization of both $\Delta H$ and $-T \Delta S$ values. 
In fact, the $\Delta H$ value of pemafibrate to PPAR $\alpha$-LBD bears a negative value $(-12.3 \mathrm{kcal} / \mathrm{mol})$ as well as other interactions, such as the interaction between PPAR $\alpha$-LBD and fenofibrate, and PPAR $\gamma$-LBD and pemafibrate. On the other hand, the $-T \Delta S$ value of pemafibrate to PPAR $\alpha$-LBD $(3.13 \mathrm{kcal} / \mathrm{mol})$ was clearly improved compared to other interactions (Table 1). The improvement of the interaction between pemafibrate and PPAR $\alpha$-LBD may be due to its two moieties: phenoxyalkyl and 2-aminobenzoxasole groups. This will be analyzed in the following section in detail. These results suggest that hydrophobic interactions, which are characteristic in the pemafibrate/PPAR $\alpha$-LBD binding, improve the affinity of pemafibrate to $\operatorname{PPAR} \alpha$.
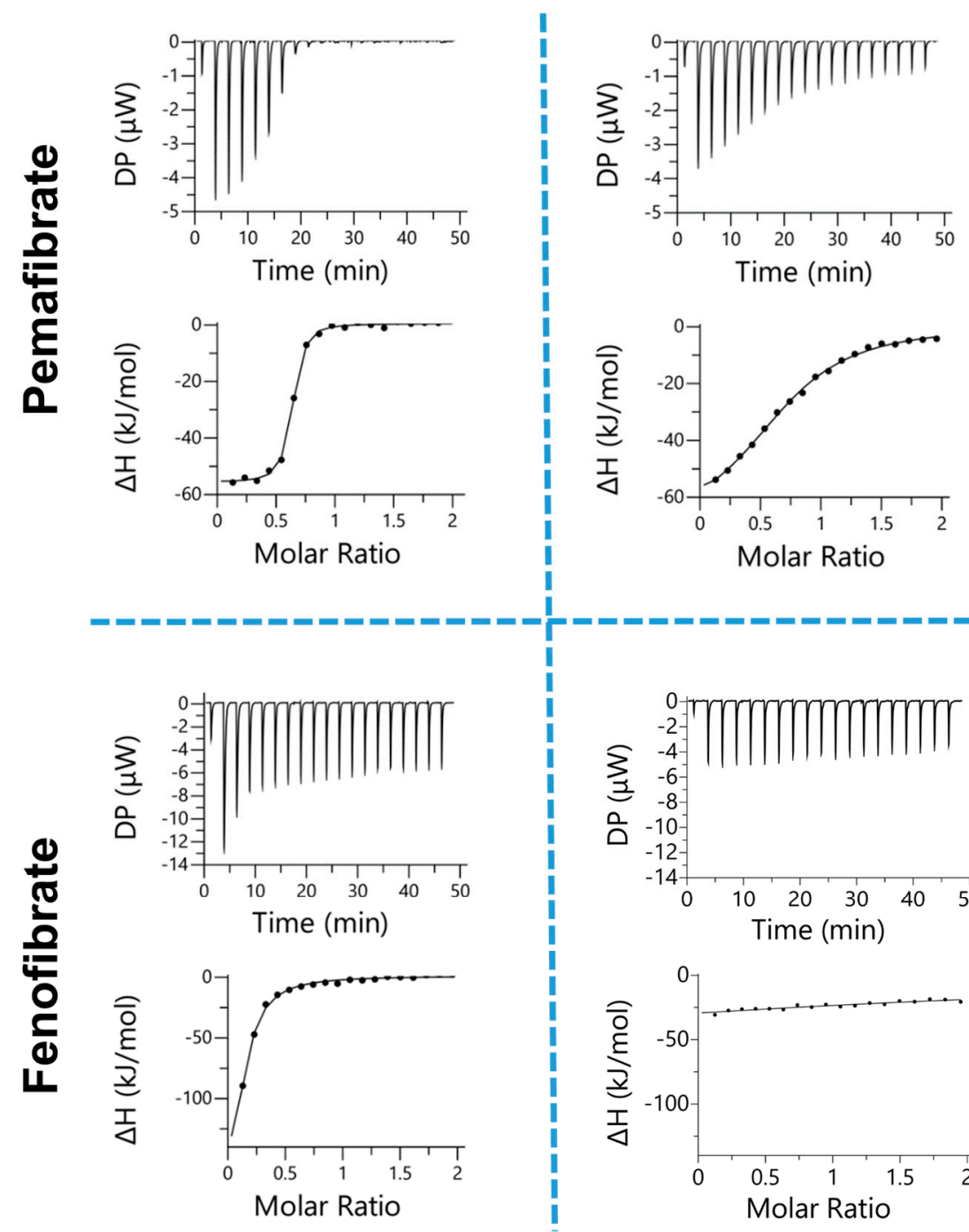

PPARa LBD

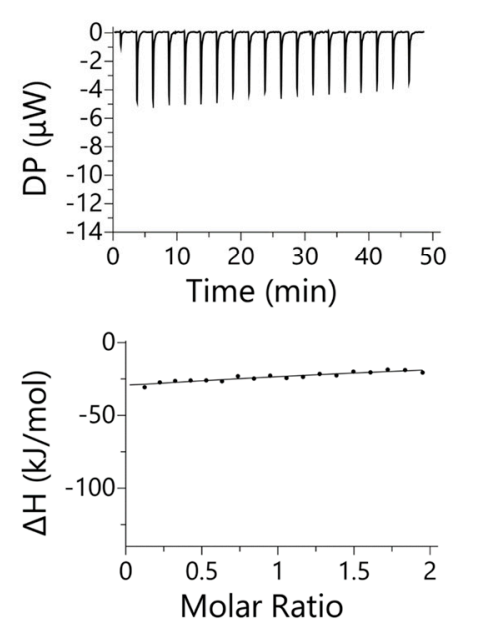

PPARY LBD

Figure 1. Isothermal titration calorimetry (ITC) analysis of fenofibric acid and pemafibrate binding to peroxisome proliferator activated receptor $\alpha(\operatorname{PPAR} \alpha)$ and PPAR $\gamma$ ligand binding domain (LBD). The upper and lower panels of ITC data represent the heat signal generated by the binding of each ligand to PPARs in the cells and integration of the signal per injection, respectively. All the relevant parameters are given in Table 1.

\subsection{Crystal Structure Analysis for Pemafibrate and SRC1 Peptide Binding form of PPAR $\alpha$-LBD}

ITC measurements have suggested that the characteristic hydrophobic interactions would improve affinity of pemafibrate to PPAR $\alpha$. To prove this point from structural analysis, crystal structure of PPAR $\alpha$-LBD/pemafibrate/SRC1 complex is determined at $3.2 \AA$ resolution (Table 2). The structure 
forms dimer in asymmetric unit, in one subunit the protein binds both with pemafibrate and the SRC1 peptide (green, Figure 2A) while in the other subunit the protein binds only with pemafibrate (orange, Figure 2A). These domains are referred as SRC1 binding domain and non-SRC1 binding domain, respectively (Figure 2).

Table 2. Statistics of X-ray diffraction data collection for PPAR $\alpha$-LBD (194-468) complexed with pemafibrate and coactivator peptide, SRC1.

\begin{tabular}{|c|c|}
\hline & $\operatorname{PPAR} \alpha-\mathrm{LBD} /$ Pemafibrate/SRC1 \\
\hline Space group & $\mathrm{P} 3{ }_{1} 21$ \\
\hline \multicolumn{2}{|l|}{ Unit cell parameters } \\
\hline a $(\AA)$ & 82.74 \\
\hline $\mathrm{b}(\AA)$ & 82.74 \\
\hline$c(\AA)$ & 177.5 \\
\hline$\alpha$ (degree) & 90.0 \\
\hline$\beta$ (degree) & 90.0 \\
\hline$\gamma$ (degree) & 120.0 \\
\hline X-ray source & $\begin{array}{c}\text { PF } \\
\text { BL5A }\end{array}$ \\
\hline Wavelength $(\AA)$ & 1.00 \\
\hline Resolution $(\AA)$ & $\begin{array}{c}45.7-3.2 \\
(3.26-3.2)\end{array}$ \\
\hline No. of reflections ${ }^{a}$ & 131,418 \\
\hline No. of unique reflections & 224,57 \\
\hline Completeness (\%) & $100(100)$ \\
\hline $\mathrm{I} / \operatorname{sig}(\mathrm{I})$ & $20.8(1.5)$ \\
\hline$R_{\text {merge }} \mathrm{b}$ & $0.080(0.678)$ \\
\hline $\mathrm{CC} 1 / 2$ & $0.996(0.800)$ \\
\hline$R^{\mathrm{c}}$ & 0.190 \\
\hline$R_{\text {free }}{ }^{\mathrm{d}}$ & 0.253 \\
\hline \multicolumn{2}{|l|}{ RMSD of geometry } \\
\hline Bond length $(\AA)$ & 0.013 \\
\hline Bond angle (degree) & 1.656 \\
\hline \multicolumn{2}{|l|}{ Geometry } \\
\hline Ramachandran outlier (\%) & 0.4 \\
\hline Ramachandran favored (\%) & 99.6 \\
\hline PDB code & 6L96 \\
\hline
\end{tabular}

a Sigma cutoff was set to none $(\mathrm{F}>0 \sigma \mathrm{F}) .{ }^{\mathrm{b}} R_{\text {merge }}=\Sigma_{h} \Sigma_{i}\left|I_{i}(h)-<I(h)>\right| / \Sigma_{h} I(h)$, where $I_{i}(h)$ is the ${ }^{\text {th }}$ measurement of reflection $h$, and $\langle I(h)>$ is the mean value of the symmetry-related reflection intensities. Values in brackets are for the shell of the highest resolution. ${ }^{c} R=\Sigma|| F_{o}|-| F_{c} \| / \Sigma\left|F_{o}\right|$, where $F_{o}$ and $F_{c}$ are the observed and calculated structure factors used in the refinement, respectively. ${ }^{d} R_{\text {free }}$ is the $R$-factor calculated using $5 \%$ of the reflections chosen at random and omitted from the refinement.

The structure comparison between SRC1 binding (Figure 2B) and non-SRC1 binding domains (Figure 2D) indicates that overall the structures are almost identical to each other; in fact, the root mean square deviation value for $\mathrm{C} \alpha$ atoms is $0.315 \AA$, which indicates that no drastic conformational change is induced in the PPAR $\alpha$-LBD structure by the binding of SRC1 peptide. In both of the structures, pemafibrate binds to the LBP of PPAR $\alpha$-LBD (Figure 2C,E). The carboxyl group of pemafibrate forms hydrogen bonding interactions with Y314, H440, and Y464, Figure 2C,E, and these interactions were also observed in other agonists bound to PPAR $\alpha[3,37,38]$. 
Here, the flexibility of pemafibrate appeared to be changed with or without binding of SRC1 peptide from structure comparison between the domains. In fact, the electron density of pemafibrate is clearly confirmed in the SRC1 binding domain (blue mesh in Figure 2C); the electron density has a Y-shaped form to fill a cavity at the LBP of PPAR $\alpha$ (Figure 2C). On the other hand, there is no electron density for phenoxyalkyl group of pemafibrate in the non-SRC1 binding domain (blue mesh in Figure 2E). The high flexibility of the phenoxyalkyl group of pemafibrate would work for favorable interactions with PPAR $\alpha$ by changing its conformation depending on the structural changes of PPAR $\alpha$ induced by the binding of various coactivators.

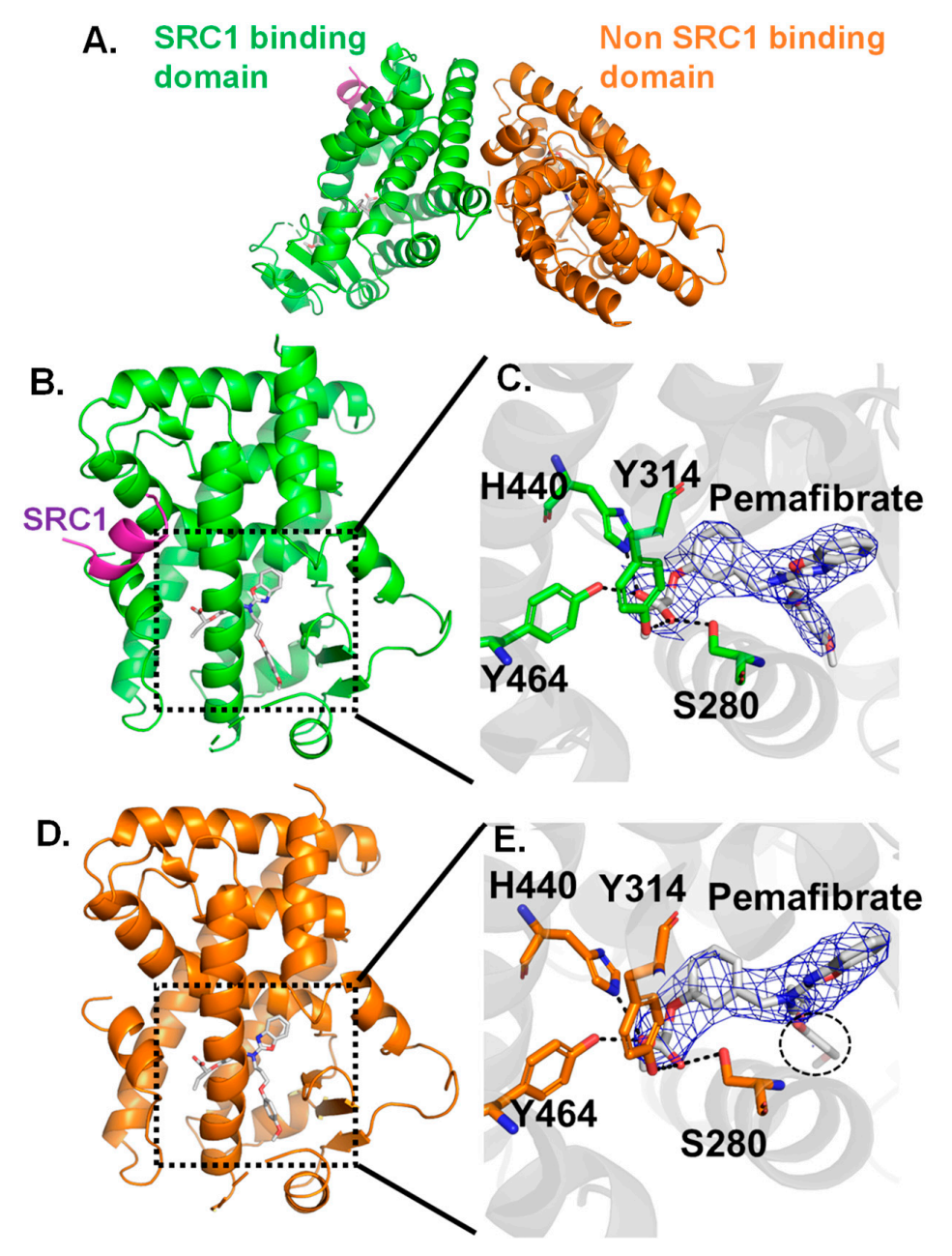

Figure 2. (A) Overall structure of $\operatorname{PPAR} \alpha-\mathrm{LBD} /$ pemafibrate/SRC1 peptide. The domain which binds both pemafibrate and SRC1 peptide colored in green, and the domain which binds only pemafibrate colored in orange. Crystal structure of SRC1 binding domain (B) and LBP site (C). Crystal structure of the non-SRC1 binding domain (D) and LBP site (E). In both structures, three residues (Y314, H440, and Y464) form hydrogen bonds with the carboxyl group of pemafibrate. The $2 F_{\mathrm{o}}-F_{\mathrm{c}}$ electron density map contoured at $1.0 \sigma$.

\subsection{Interaction Energy Analysis between Pemafibrate and PPAR $\alpha$-LBD Based on FMO Method}

Utilizing the crystal structure of PPAR $\alpha$-LBD/pemafibrate/SRC1 complex, residue-level interaction energies between pemafibrate and residues at LBP of PPAR $\alpha$ can be quantitatively obtained through computational analysis. In this study, the quantum-mechanical (electron correlation incorporated) FMO method has been used to calculate the interaction energies. The residue-level interaction energies are estimated through interfragment interaction energy (IFIE) calculations. The calculated IFIEs consist of three energy terms namely, HF-IFIEs, dMP2-IFIEs, and MP2-IFIEs. HF-IFIEs and MP2-IFIEs are obtained 
by applying the Hartree-Fock and Møller-Plesset perturbation theory, respectively. HF-IFIEs mostly represent electrostatic interactions. MP2-IFIEs are the sum of HF-IFIEs and electron correlation energies, which roughly represent hydrophobic interactions. The dMP2-IFIEs are obtained by subtracting HF-IFIEs from MP2-IFIEs; thus, the term dMP2-IFIEs contains only the electron correlation energies.

For the convenience to analyze IFIEs, the LBP of PPAR $\alpha$-LBD is divided into three sites depending on the binding of pemafibrate: site A (red surface in Figure 3A), site B (green surface in Figure 3A), and site $C$ (orange surface in Figure $3 \mathrm{~A}$ ), respectively. The three sites are formed by the residues indicated in Figure 3B. Among the six residues forming site A, five residues bear polar groups on their side chains (Figure 3B), suggesting that electrostatic interactions would be formed at site A with pemafibrate. On the other hand, site B and C are mainly formed by hydrophobic residues (eight out of ten residues), inferring that, at these sites, hydrophobic interactions would be the main contributor to form stable interactions with pemafibrate (Figure 3B).
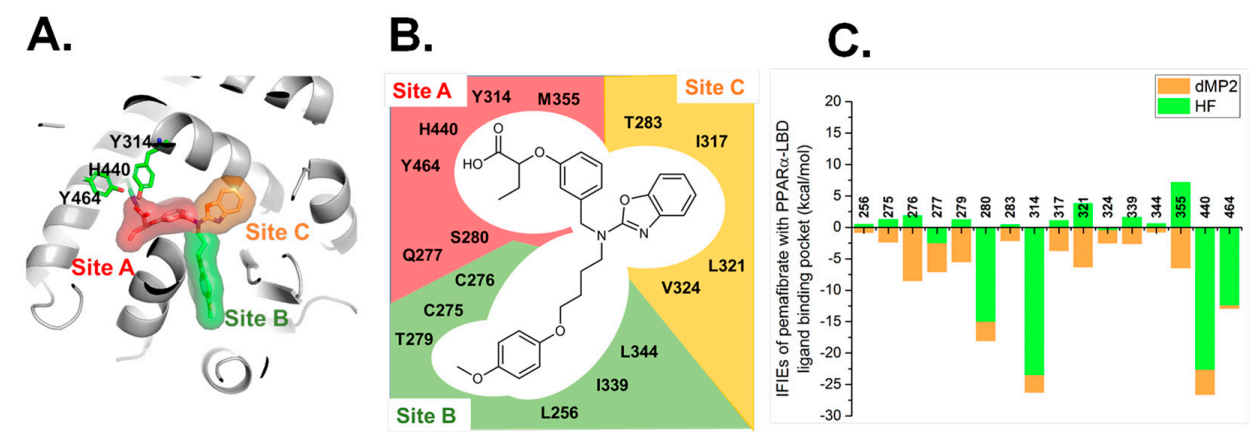

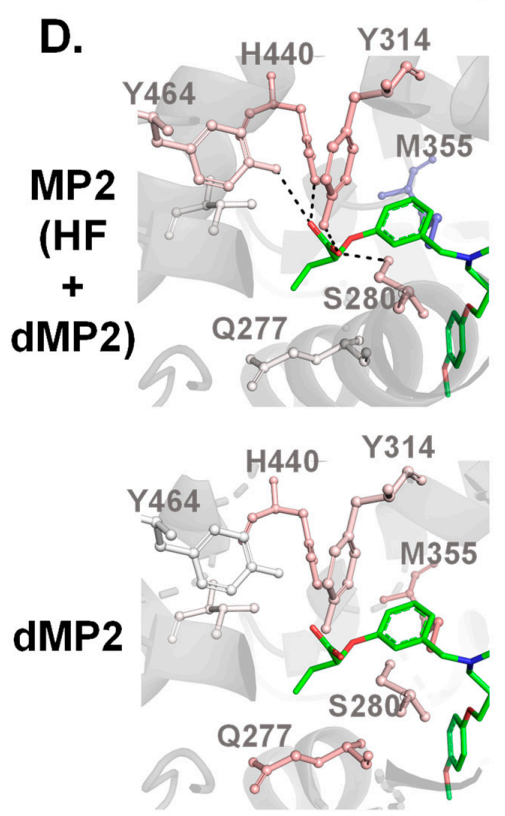

Site A
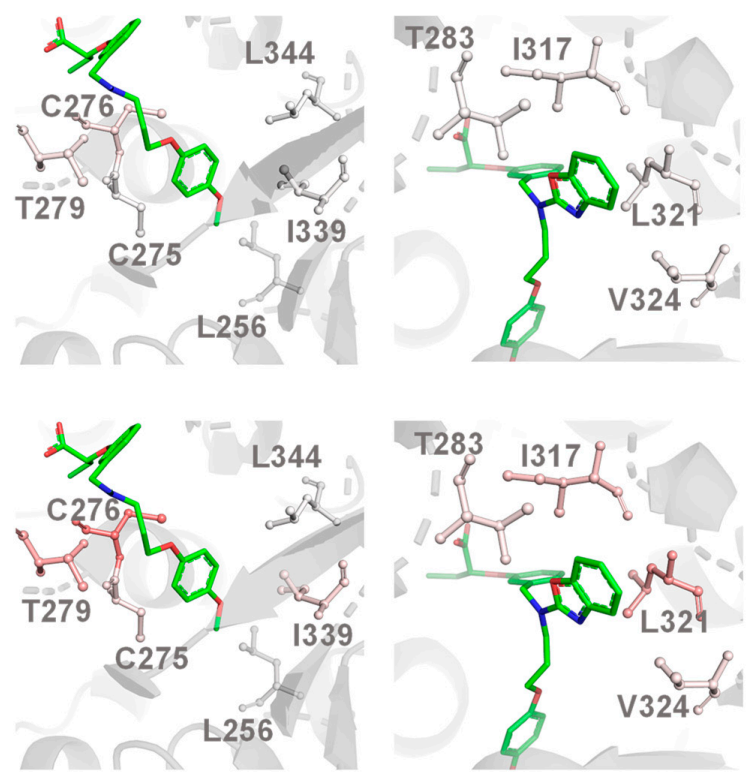

Site B

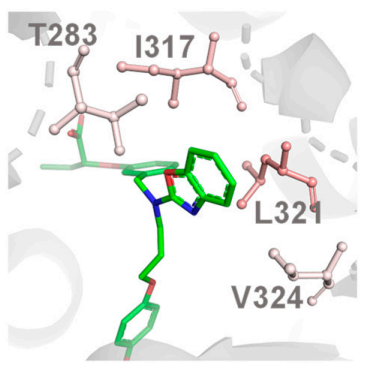

Site C

Figure 3. (A) Division of the LBP site of PPAR $\alpha$ into three sites. The LBP is divided into three sites: site A (red surface), site B (green surface), and site C (orange surface). (B) Potential interacting residues at the LBP of PPAR $\alpha$ with pemafibrate. A total of 16 residues were located within $3.2 \AA$ distance from pemafibrate. (C) Significant interfragment interaction energies (IFIEs) between pemafibrate and LBP residues of PPAR $\alpha$. The HF-IFIEs and dMP2-IFIEs represented in green and orange, respectively. (D) Representation of MP2-IFIEs (upper row) and dMP2-IFIEs (bottom row) at the three sites on the crystal structure of the PPAR $\alpha$-LBD/pemafibrate/SRC1 peptide. The structures are colored depending on the magnitude of the IFIEs value. Positive (repulsive) and negative (attractive) IFIEs are colored by blue and red, respectively. 
We calculated IFIEs that represent interaction energies between pemafibrate and residues at LBP of PPAR $\alpha$-LBD to show which residues interact with pemafibrate strongly (Figure 3C). In addition, depending on the magnitude of MP2-IFIEs and dMP2-IFIEs, the residues were colored on crystal structure of PPAR $\alpha-\mathrm{LBD} /$ pemafibrate/SRC1. The figures would be helpful in figuring out the residues that interact with pemafibrate visually. As expected, four residues at site A (S280, Y314, Y440, and Y464) formed strong electrostatic interactions with pemafibrate compared with other residues (Figure 3D, MP2 at the site A). In fact, about $65 \%$ of the total MP2-IFIEs $(-113.20 \mathrm{kcal} / \mathrm{mol})$ are derived from HF-IFIEs of these four residues $(-73.55 \mathrm{kcal} / \mathrm{mol}$ ) (Figure 3C). The remaining 35\% of stabilization would be brought by the hydrophobic interaction (dMP2-IFIEs) between pemafibrate and PPAR $\alpha$-LBD. Evidently, almost all of the residues at the three sites are colored in red when the contribution of dMP2-IFIEs was reflected in the structure (lower line in Figure 3D). This suggested that the accumulation of weak hydrophobic interactions between pemafibrate and hydrophobic residues at the LBP of PPAR $\alpha$ also contribute to improving the binding affinity.

\section{Discussion}

Compared with fenofibrate, the administration of a lower concentration of pemafibrate strongly and selectively activates PPAR $\alpha$. By using the results derived through the combination of analysis of the crystal structure, ITC measurement, and FMO calculations in this study, we propose two plausible reasons for the novel SPPARM $\alpha$ activity of pemafibrate in detail (Figure 4).

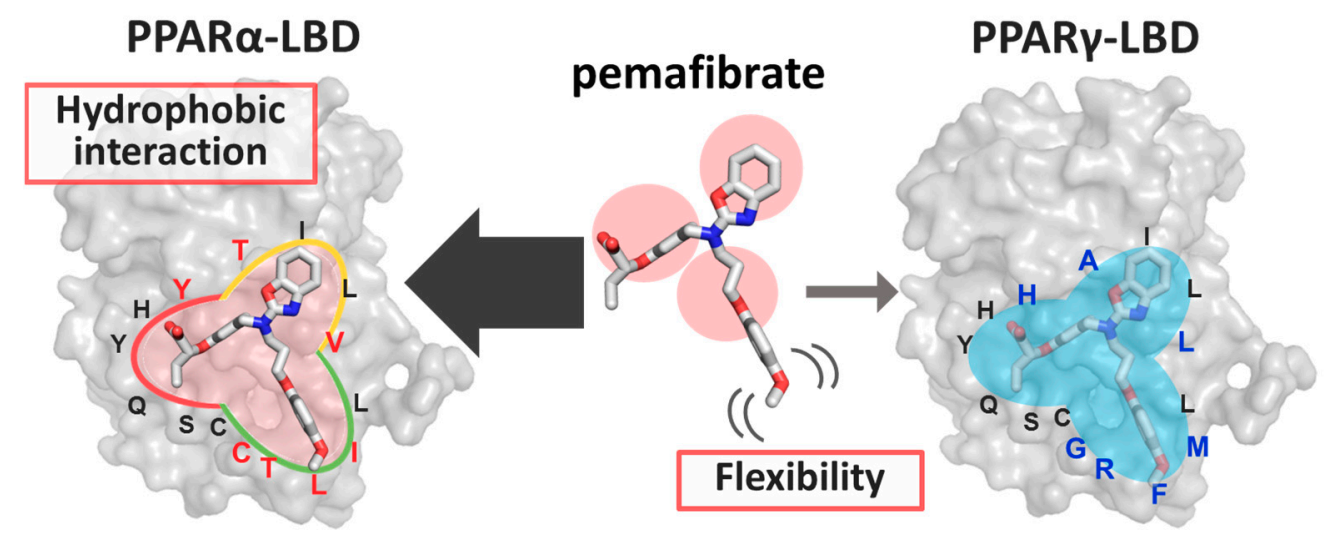

Figure 4. Schematic view of the molecular mechanism behind pemafibrate as a selective PPAR $\alpha$ modulator (SPPARM $\alpha)$.

The first reason is that the accumulation of hydrophobic interactions formed between several hydrophobic residues with pemafibrate could improve the overall binding affinity between pemafibrate and PPAR $\alpha$. Crystal structure analysis indicated that chemical groups of pemafibrate occupy three sites (site A, site B, and site C) at LBP of PPAR $\alpha$ (Figure 2C), whereas fenofibrate can bind to PPAR $\alpha$ by occupying two sites from their chemical structure. The difference in the number of interactions would be a reason that pemafibrate works as a strong SPPARM $\alpha$ compared with fenofibrate. Furthermore, this point is well supported by the FMO analysis; around 16 residues at the LBP of PPAR $\alpha$ (Figure 3B) form weak hydrophobic interactions with pemafibrate (see dMP2 values in Figure 3C). The results indicate that one-point mutation at the LBP would have only little effect on the total binding affinity of pemafibrate because other residues would compensate for the loss of the interaction by the mutation. It should be mentioned here that our earlier study indicated that, for the case of pemafibrate, five PPAR $\alpha$ mutants (C275A, S280A, L321A, I339A, and L344A) remained active (range of 25 to 70\%) compared with wild type PPAR $\alpha$ [33]. On the other hand, for the case of fenofibric acid, the activity was drastically decreased by the mutations, especially for the I339A and L344A mutants, the activity was hardly detected [33]. Meanwhile, comparing LBP structures of PPAR $\alpha$ and PPAR $\gamma$ indicates their sequence differences: C275, T279, T283, Y314, V324, and I339 in PPAR $\alpha$ are mutated to Gly, Arg, Ala, 
His, Leu, and Met in PPAR $\gamma$, respectively (colored residues in Figure 4). These differences would explain the reduction in the binding affinity of pemafibrate to PPAR $\gamma$. Of course, we recognize that the activity change was measured only for Ala mutants of PPAR $\alpha$ [33]. Thus, we cannot evaluate another possibility on how pemafibrate works as an SPPARM $\alpha$-less favorable interactions, such as steric clash of pemafibrate formed with PPAR $\gamma$ and not with PPAR $\alpha$ affects the binding affinity. In the future, the activity measurement for PPAR $\alpha$ mutants of which residues at the LBP are mutated corresponding to the LBP of PPAR $\gamma$ or vice versa should be analyzed to estimate this point.

The second and more important reason is that pemafibrate can change their conformation due to its flexible phenoxyalkyl group and can make suitable interactions with PPAR $\alpha$ (Figure 4). The X-ray structure analysis indicates that electron density map for site A and site B of pemafibrate is clearly observed with or without the binding of SRC1 peptide (Figure 2C,E), whereas the electron density for the site $\mathrm{C}$ is disappeared in the absence of the peptide (Figure 2E). By referring to some studies, the characteristic conformational change of PPAR $\alpha$ would be induced by the binding of coactivator. Representation is the conformational change at the AF2 interface and $\Omega$-loop. Especially, the physiological importance of the conformational change at $\Omega$-loop is reported for now. In fact, we have indicated earlier that the I272A mutation, which is one of the residues near the $\Omega$-loop completely lost its activity [33]. Notably, the phenoxyalkyl group of pemafibrate is at site B, which includes the $\Omega$-loop. The high flexibility at the phenoxyalkyl group may enable pemafibrate to bind to PPAR $\alpha$ strongly with an "induced-fit" mechanism in response to structural changes at site B induced by binding of coactivators. Future works on several mutations of PPAR $\alpha$ would help to figure out the mechanism of how pemafibrate can keep this network as a SPPARM $\alpha$.

\section{Conclusions}

In this study, we succeeded to determine the crystal structure of PPAR $\alpha$-LBD/pemafibrate/SRC1, which indicates that pemafibrate fully occupies the cavity at the LBP of PPAR $\alpha$. The ITC and FMO analysis indicate a strong potency of pemafibrate to PPAR $\alpha$ appeared to be brought by the accumulation of weak hydrophobic interactions between the residues at the LBP of PPAR $\alpha$ and pemafibrate; this point supports our earlier hypothesis made through combinational approaches of docking simulation and FMO analysis of PPAR $\alpha$-LBD and pemafibrate [33]. The study has also revealed that phenoxyalkyl group of pemafibrate is highly flexible when no coactivator is bound with pemafibrate/PPAR $\alpha$. The binding of the coactivator fixes the conformation of the phenoxyalkyl group as indicated in the crystal structure analysis. This flexible part of the pemafibrate could contribute to the induced-fit feature of the pemafibrate/PPAR $\alpha$ complex [33] and the potential coactivator-dependent activation as SPPARM $\alpha$. These results suggest that the optimum interaction between the drug molecule and the target protein and the flexibility of the molecule is important for the unique features of this novel SPPARM $\alpha$. It also highlights the usefulness of FMO calculations for predicting the molecular structure of the related functions even before the verification by $\mathrm{X}$-ray structure analysis.

\section{Materials and Methods}

\subsection{Preparation of PPARs Ligands and Coactivator Peptides}

Pemafibrate was provided by Kowa Corporation (Tokyo, Japan). Fenofibric acid was purchased from Tokyo Chemical Industry (Nagoya, Aichi, Japan). These compounds were dissolved into DMSO. The coactivator peptide, SRC1 (687-HKILHRLLQEGS-698), was synthesized by GeneScript (Piscataway, NJ, USA). The peptide was used to perform crystallization and ITC measurement.

\subsection{Overexpression and Purification of PPAR $\alpha$ LBD and PPAR $\gamma$ LBD}

A plasmid containing human PPAR $\alpha$ LBD (residues 194-468) was cloned into the pET28 vector. These plasmids were transformed to BL21 (DE3) strain. Escherichia coli (E. coli) cells were cultured at $37^{\circ} \mathrm{C}$ in LB medium with $30 \mu \mathrm{g} / \mathrm{mL}$ Kanamycin Sulfate. After that, PPAR $\alpha$ LBD expression was 
induced by adding $0.5 \mathrm{mM}$ of isopropyl $\beta$-D-thiogalactopyranoside to the medium and incubated for $48 \mathrm{~h}$ at $16^{\circ} \mathrm{C}$. These cells were collected and resuspended into buffer A ( $20 \mathrm{mM} \mathrm{HEPES-NaOH} \mathrm{(pH} \mathrm{8.0),}$ $100 \mathrm{mM} \mathrm{NaCl}, 0.5 \mathrm{mM}$ Tris(2-carboxyethyl)phosphine (TCEP), and 5\% (v/v) glycerol). After sonication, the insoluble fraction was removed by centrifugation at $10,000 \times g$ for $30 \mathrm{~min}$. The obtained supernatant was applied to a HisTrap HP column (GE Healthcare) equilibrated with buffer A. The column was washed by buffer A containing $70 \mathrm{mM}$ imidazole. The samples were eluted with buffer A containing $300 \mathrm{mM}$ imidazole. These eluted fractions were concentrated and his-tag was removed by $200 \mathrm{U}$ thrombin, and dialyzed in buffer B (20 mM HEPES-NaOH (pH 8.0), $10 \mathrm{mM} \mathrm{NaCl}, 0.5 \mathrm{mM}$ TCEP, and $5 \%(v / v)$ glycerol) using a molecular porous membrane (RERPLIGEN) at $4{ }^{\circ} \mathrm{C}$ for two days. Dialyzed samples were concentrated and applied to a MonoQ column (GE Healthcare, Chicago, IL, USA) and eluted with an $\mathrm{NaCl}$ gradient $(10-500 \mathrm{mM})$. The eluted fraction was further purified on a gel filtration column (Superdex 200 Increase) (GE Healthcare) equilibrated with bufferA. Fractions containing samples were collected and applied to an SDS-PAGE to check their purity. The samples were utilized in subsequent experiments. Human PPAR $\gamma$ LBD (residues 206-477) were cloned into pET28 vector, and the proteins were expressed and purified by the same procedure described in PPAR $\alpha$ LBD.

\subsection{Crystallization and X-ray Data Collection of the PPAR $\alpha$-LBD/Pemafibrate/SRC1 Peptide}

The purified PPAR $\alpha$-LBD samples were concentrated to about $15 \mathrm{mg} / \mathrm{mL}$. The samples which ligate pemafibrate and SRC1 peptide (PPAR $\alpha$-LBD/pemafibrate/SRC1) were obtained by mixing PPAR $\alpha$-LBD, pemafibrate and SRC1 peptide as the following molar ratio: 1:3:5 = PPAR $\alpha$-LBD:pemafibrate:SRC1 peptide. The mixed samples were incubated for $24 \mathrm{~h}$ at $4{ }^{\circ} \mathrm{C}$. The crystals of the PPAR $\alpha$-LBD/pemafibrate/SRC1 appeared under the condition of the following reservoir: $1.2 \mathrm{M}$ ammonium sulfate and $0.1 \mathrm{M}$ bis-tris- $\mathrm{HCl}(\mathrm{pH} 6.5)$ at $22{ }^{\circ} \mathrm{C}$.

The obtained crystals were soaked into cryo-reservoir containing 20\% (v/v) glycerol and $100 \mu \mathrm{M}$ pemafibrate in the reservoir solution. The soaked crystals were mounted and flash-cooled under a nitrogen stream $\left(-173^{\circ} \mathrm{C}\right)$. Diffraction data were collected using Dectris Pilatus3 S6M detector at BL5A of Photon Factory (Tsukuba, Japan). The data were collected, scaled, and integrated by HKL2000 and SCALEPACK [39]. The initial phase was determined by the molecular replacement method with MOLREP [40] utilizing the structure for chain A of the complex PPAR $\alpha$ /AL26-29 (PDB ID: 5 HYK). Model building and structure refinement were performed by COOT [41] and REFMAC [42], respectively. All figures were prepared by PyMOL [43]. The crystallographic table is written in Table 2.

\subsection{Fragment Molecular Orbital Calculations}

The fragment molecular orbital (FMO) calculation for PPAR $\alpha$-LBD/pemafibrate/SRC1 complex was performed by referring to the previous study [44-46]. The PPAR $\alpha$-LBD/pemafibrate/SRC1 structure was protonated at $\mathrm{pH} 7.0$ condition by the Protonate3 tool implemented in MOE [47]. Energy minimization was performed by imposing restraint on all atoms in the structure except for the hydrogen atom with molecular mechanics calculations utilizing the Amber10:EHT force-field with solvation energy accounted via the Born model with root mean square deviation (RMSD) gradient setting 0.01 $\mathrm{kcal} \mathrm{mol}^{-1} \AA^{-2}$. By referring to a previous study [48], the structure was divided into one-residue fragments. The fragment assignment and parallelized ab initio calculation system based on FMO (PAICS) input generation was done using the free utility PaicsView. The FMO calculation was performed with the PAICS software [49] at the resolution of the identity approximation of the second-order Møller-Plesset perturbation theory (RI-MP2), with the double zeta set of the correlation-consistent polarized valence basis set (cc-pVDZ) level. In this study, we analyzed the interactions between pemafibrate and PPAR $\alpha$-LBD by calculating interfragment interaction energies (IFIEs). The IFIEs were divided into three energy terms: $\mathrm{HF}$, dMP2, and MP2. Here, the counterpoise (CP) correction method [50] was adopted to calculate the IFIEs to avoid the basis set superposition error [51]. All IFIEs were CP-corrected values. The IFIEs between pemafibrate and PPAR $\alpha$-LBD were colored by the RbAnalysisFMO toolkit [52]. 


\subsection{Isothermal Titration Calorimetry}

The interaction energy between proteins (PPAR $\alpha$ LBD, PPAR $\gamma$ LBD) and ligands (pemafibrate, fenofibric acid) were estimated by the ITC experiment. Proteins and ligands were resuspended in bufferA containing $5 \%(v / v)$ DMSO. The experiment was performed at $25^{\circ} \mathrm{C}$ temperature by PEAQ-ITC (Malvern Panalytical Worcestershire, UK). To quantify the interaction energies between proteins and the pemafibrate, the final concentration of the protein and pemafibrate was determined as the following: $500 \mu \mathrm{M}$ pemafibrate filled in the syringe were titrated into 49-50 $\mu \mathrm{M}$ PPAR $\alpha$ LBD, and $50 \mu \mathrm{M}$ PPAR $\gamma$ LBD in the cell, respectively. In the case of quantification of interaction energies between proteins and the fenofibric acid, the final concentration of the protein and fenofibric acid was set to the following: 500-650 $\mu \mathrm{M}$ fenofibric acid filled in the syringe were titrated into 65-100 $\mu \mathrm{M}$ PPAR $\alpha$ LBD, 300-650 $\mu \mathrm{M}$ fenofibric acid filled in the syringe were titrated into 65-100 $\mu \mathrm{M}$ PPAR $\gamma$ LBD in the cell, respectively. The obtained data were analyzed by utilizing the PEAQ-ITC Analysis software (Malvern Panalytical).

Author Contributions: M.K. and A.K. contributed equally to this manuscript. H.T., S.N., and H.S. designed the research and managed the projects. M.K. and A.K. purified PPARs samples and performed ITC measurement. S.N. performed X-ray crystallography. Y.Y., S.A. and H.T. performed FMO calculations. M.K., A.K., Y.Y., S.A., S.I., Y.N., H.T., S.N., and H.S. analyzed and discussed the data. M.K., S.N., and H.S. wrote the manuscript. All authors have read and agreed to the published version of the manuscript.

Funding: This work was supported by AMED-CREST, Japan Agency for Medical Research Development (to H.S.), JSPS KAKENHI Grant Number 16K18688, and 18K14391 (for S.N.).

Acknowledgments: X-ray data were collected at the synchrotron facilities of the Photon Factory (PF) using beamlines BL5A (proposal No. 2018G006). The computations were performed using Research Center for Computational Science, Okazaki, Japan.

Conflicts of Interest: The authors declare no conflict of interest.

\section{Abbreviations}

$\begin{array}{ll}\text { PPAR } & \text { Peroxisome proliferator-activated receptor } \\ \text { LBD } & \text { Ligand binding domain } \\ \text { LBP } & \text { Ligand binding pocket } \\ \text { SPPARM } & \text { Selective PPAR modulator } \\ \text { FMO } & \text { Fragment molecular orbital } \\ \text { ITC } & \text { Isothermal titration calorimetry } \\ \text { IFIE } & \text { Interfragment interaction energy }\end{array}$

\section{References}

1. Willson, T.M.; Brown, P.J.; Sternbach, D.D.; Henke, B.R. The PPARs: From orphan receptors to drug discovery. J. Med. Chem. 2000, 43, 527-550. [CrossRef] [PubMed]

2. Xu, J.; Xiao, G.; Trujillo, C.; Chang, V.; Blanco, L.; Joseph, S.B.; Bassilian, S.; Saad, M.F.; Tontonoz, P.; Lee, W.N.; et al. Peroxisome proliferator-activated receptor alpha (PPARalpha) influences substrate utilization for hepatic glucose production. J. Biol. Chem. 2002, 277, 50237-50244. [CrossRef] [PubMed]

3. Bernardes, A.; Souza, P.C.; Muniz, J.R.; Ricci, C.G.; Ayers, S.D.; Parekh, N.M.; Godoy, A.S.; Trivella, D.B.; Reinach, P.; Webb, P.; et al. Molecular mechanism of peroxisome proliferator-activated receptor alpha activation by WY14643: A new mode of ligand recognition and receptor stabilization. J. Mol. Biol. 2013, 425, 2878-2893. [CrossRef]

4. Cronet, P.; Petersen, J.F.; Folmer, R.; Blomberg, N.; Sjoblom, K.; Karlsson, U.; Lindstedt, E.L.; Bamberg, K. Structure of the PPARalpha and -gamma ligand binding domain in complex with AZ 242; ligand selectivity and agonist activation in the PPAR family. Structure 2001, 9, 699-706. [CrossRef]

5. Jang, J.Y.; Bae, H.; Lee, Y.J.; Choi, Y.I.; Kim, H.J.; Park, S.B.; Suh, S.W.; Kim, S.W.; Han, B.W. Structural Basis for the Enhanced Anti-Diabetic Efficacy of Lobeglitazone on PPARgamma. Sci. Rep. 2018, 8, 31. [CrossRef] [PubMed] 
6. Mosure, S.A.; Shang, J.; Eberhardt, J.; Brust, R.; Zheng, J.; Griffin, P.R.; Forli, S.; Kojetin, D.J. Structural Basis of Altered Potency and Efficacy Displayed by a Major in Vivo Metabolite of the Antidiabetic PPARgamma Drug Pioglitazone. J. Med. Chem. 2019, 62, 2008-2023. [CrossRef]

7. Ebdrup, S.; Pettersson, I.; Rasmussen, H.B.; Deussen, H.J.; Frost Jensen, A.; Mortensen, S.B.; Fleckner, J.; Pridal, L.; Nygaard, L.; Sauerberg, P. Synthesis and biological and structural characterization of the dual-acting peroxisome proliferator-activated receptor alpha/gamma agonist ragaglitazar. J. Med. Chem. 2003, 46, 1306-1317. [CrossRef]

8. Oyama, T.; Toyota, K.; Waku, T.; Hirakawa, Y.; Nagasawa, N.; Kasuga, J.I.; Hashimoto, Y.; Miyachi, H.; Morikawa, K. Adaptability and selectivity of human peroxisome proliferator-activated receptor (PPAR) pan agonists revealed from crystal structures. Acta Crystallogr. D Biol. Crystallogr. 2009, 65, 786-795. [CrossRef]

9. Kuwabara, N.; Oyama, T.; Tomioka, D.; Ohashi, M.; Yanagisawa, J.; Shimizu, T.; Miyachi, H. Peroxisome proliferator-activated receptors (PPARs) have multiple binding points that accommodate ligands in various conformations: Phenylpropanoic acid-type PPAR ligands bind to PPAR in different conformations. depending on the subtype. J. Med. Chem. 2012, 55, 893-902. [CrossRef]

10. Xu, H.E.; Lambert, M.H.; Montana, V.G.; Plunket, K.D.; Moore, L.B.; Collins, J.L.; Oplinger, J.A.; Kliewer, S.A.; Gampe, R.T., Jr.; McKee, D.D.; et al. Structural determinants of ligand binding selectivity between the peroxisome proliferator-activated receptors. Proc. Natl. Acad. Sci. USA 2001, 98, 13919-13924. [CrossRef]

11. Fruchart, J.C. Selective peroxisome proliferator-activated receptor alpha modulators (SPPARMalpha): The next generation of peroxisome proliferator-activated receptor alpha-agonists. Cardiovasc. Diabetol. 2013, 12, 82. [CrossRef] [PubMed]

12. Yew, T.; Toh, S.A.; Millar, J.S. Selective peroxisome proliferator-activated receptor-gamma modulation to reduce cardiovascular risk in patients with insulin resistance. Recent Pat. Cardiovasc. Drug Discov. 2012, 7, 33-41. [CrossRef] [PubMed]

13. Einstein, M.; Akiyama, T.E.; Castriota, G.A.; Wang, C.F.; McKeever, B.; Mosley, R.T.; Becker, J.W.; Moller, D.E.; Meinke, P.T.; Wood, H.B.; et al. The differential interactions of peroxisome proliferator-activated receptor gamma ligands with Tyr473 is a physical basis for their unique biological activities. Mol. Pharmacol. 2008, 73, 62-74. [CrossRef] [PubMed]

14. Mukherjee, R.; Hoener, P.A.; Jow, L.; Bilakovics, J.; Klausing, K.; Mais, D.E.; Faulkner, A.; Croston, G.E.; Paterniti, J.R., Jr. A selective peroxisome proliferator-activated receptor-gamma (PPARgamma) modulator blocks adipocyte differentiation but stimulates glucose uptake in 3T3-L1 adipocytes. Mol. Endocrinol. 2000, 14, 1425-1433.

15. Higgins, L.S.; Depaoli, A.M. Selective peroxisome proliferator-activated receptor gamma (PPARgamma) modulation as a strategy for safer therapeutic PPARgamma activation. Am. J. Clin. Nutr. 2010, 91, 267S-272S. [CrossRef]

16. Sugiyama, Y.; Shimura, Y.; Ikeda, H. Effects of pioglitazone on hepatic and peripheral insulin resistance in Wistar fatty rats. Arzneimittelforschung 1990, 40, 436-440.

17. Young, P.W.; Buckle, D.R.; Cantello, B.C.; Chapman, H.; Clapham, J.C.; Coyle, P.J.; Haigh, D.; Hindley, R.M.; Holder, J.C.; Kallender, H.; et al. Identification of high-affinity binding sites for the insulin sensitizer rosiglitazone (BRL-49653) in rodent and human adipocytes using a radioiodinated ligand for peroxisomal proliferator-activated receptor gamma. J. Pharmacol. Exp. Ther. 1998, 284, 751-759.

18. Tontonoz, P.; Hu, E.; Graves, R.A.; Budavari, A.I.; Spiegelman, B.M. mPPAR gamma 2: Tissue-specific regulator of an adipocyte enhancer. Genes Dev. 1994, 8, 1224-1234. [CrossRef]

19. Alagona, P., Jr. Fenofibric acid: A new fibrate approved for use in combination with statin for the treatment of mixed dyslipidemia. Vasc. Health Risk Manag. 2010, 6, 351-362. [CrossRef]

20. Tenenbaum, A.; Fisman, E.Z. Balanced pan-PPAR activator bezafibrate in combination with statin: Comprehensive lipids control and diabetes prevention? Cardiovasc. Diabetol. 2012, 11, 140. [CrossRef]

21. Fazio, S.; Linton, M.F. The role of fibrates in managing hyperlipidemia: Mechanisms of action and clinical efficacy. Curr. Atheroscler. Rep. 2004, 6, 148-157. [CrossRef] [PubMed]

22. Remick, J.; Weintraub, H.; Setton, R.; Offenbacher, J.; Fisher, E.; Schwartzbard, A. Fibrate therapy: An update. Cardiol. Rev. 2008, 16, 129-141. [CrossRef] [PubMed]

23. Boissonnat, P.; Salen, P.; Guidollet, J.; Ferrera, R.; Dureau, G.; Ninet, J.; Renaud, S.; de Lorgeril, M. The long-term effects of the lipid-lowering agent fenofibrate in hyperlipidemic heart transplant recipients. Transplantation 1994, 58, 245-247. [CrossRef] [PubMed] 
24. Duez, H.; Lefebvre, B.; Poulain, P.; Torra, I.P.; Percevault, F.; Luc, G.; Peters, J.M.; Gonzalez, F.J.; Gineste, R.; Helleboid, S.; et al. Regulation of human apoA-I by gemfibrozil and fenofibrate through selective peroxisome proliferator-activated receptor alpha modulation. Arterioscler. Thromb. Vasc. Biol. 2005, 25, 585-591. [CrossRef]

25. Robins, S.J. PPARalpha ligands and clinical trials: Cardiovascular risk reduction with fibrates. J. Cardiovasc. Risk 2001, 8, 195-201. [CrossRef]

26. Mychaleckyj, J.C.; Craven, T.; Nayak, U.; Buse, J.; Crouse, J.R.; Elam, M.; Kirchner, K.; Lorber, D.; Marcovina, S.; Sivitz, W.; et al. Reversibility of fenofibrate therapy-induced renal function impairment in ACCORD type 2 diabetic participants. Diabetes Care 2012, 35, 1008-1014. [CrossRef]

27. Yamazaki, Y.; Abe, K.; Toma, T.; Nishikawa, M.; Ozawa, H.; Okuda, A.; Araki, T.; Oda, S.; Inoue, K.; Shibuya, K.; et al. Design and synthesis of highly potent and selective human peroxisome proliferator-activated receptor alpha agonists. Bioorg. Med. Chem. Lett. 2007, 17, 4689-4693. [CrossRef]

28. Fruchart, J.C. Pemafibrate (K-877). a novel selective peroxisome proliferator-activated receptor alpha modulator for management of atherogenic dyslipidaemia. Cardiovasc. Diabetol. 2017, 16, 124. [CrossRef]

29. Raza-Iqbal, S.; Tanaka, T.; Anai, M.; Inagaki, T.; Matsumura, Y.; Ikeda, K.; Taguchi, A.; Gonzalez, F.J.; Sakai, J.; Kodama, T. Transcriptome Analysis of K-877 (a Novel Selective PPARalpha Modulator (SPPARMalpha))Regulated Genes in Primary Human Hepatocytes and the Mouse Liver. J. Atheroscler. Thromb. 2015, 22, 754-772. [CrossRef]

30. Ishibashi, S.; Yamashita, S.; Arai, H.; Araki, E.; Yokote, K.; Suganami, H.; Fruchart, J.C.; Kodama, T. Effects of K-877. a novel selective PPARalpha modulator (SPPARMalpha), in dyslipidaemic patients: A randomized, double blind, active- and placebo-controlled, phase 2 trial. Atherosclerosis 2016, 249, 36-43. [CrossRef]

31. Yokote, K.; Yamashita, S.; Arai, H.; Araki, E.; Suganami, H.; Ishibashi, S. A Pooled Analysis of Pemafibrate Phase II/III Clinical Trials Indicated Significant Improvement in Glycemic and Liver Function-related Parameters. Atheroscler. Suppl. 2018, 32, 154-155. [CrossRef]

32. Arai, H.; Yamashita, S.; Yokote, K.; Araki, E.; Suganami, H.; Ishibashi, S. Efficacy and safety of K-877. a novel selective peroxisome proliferator-activated receptor alpha modulator (SPPARMalpha), in combination with statin treatment: Two randomised, double-blind, placebo-controlled clinical trials in patients with dyslipidaemia. Atherosclerosis 2017, 261, 144-152. [CrossRef] [PubMed]

33. Yamamoto, Y.; Takei, K.; Arulmozhiraja, S.; Sladek, V.; Matsuo, N.; Han, S.I.; Matsuzaka, T.; Sekiya, M.; Tokiwa, T.; Shoji, M.; et al. Molecular association model of PPARalpha and its new specific and efficient ligand. pemafibrate: Structural basis for SPPARMalpha. Biochem. Biophys. Res. Commun. 2018, 499, 239-245. [CrossRef] [PubMed]

34. Hennuyer, N.; Duplan, I.; Paquet, C.; Vanhoutte, J.; Woitrain, E.; Touche, V.; Colin, S.; Vallez, E.; Lestavel, S.; Lefebvre, P.; et al. The novel selective PPARalpha modulator (SPPARMalpha) pemafibrate improves dyslipidemia. enhances reverse cholesterol transport and decreases inflammation and atherosclerosis. Atherosclerosis 2016, 249, 200-208. [CrossRef]

35. Freire, E. Do enthalpy and entropy distinguish first in class from best in class? Drug Discov. Today 2008, 13, 869-874. [CrossRef]

36. Chaires, J.B. Calorimetry and thermodynamics in drug design. Annu. Rev. Biophys. 2008, 37, $135-151$. [CrossRef]

37. Egawa, D.; Itoh, T.; Akiyama, Y.; Saito, T.; Yamamoto, K. 17-OxoDHA Is a PPARalpha/gamma Dual Covalent Modifier and Agonist. ACS Chem. Biol. 2016, 11, 2447-2455. [CrossRef]

38. Jin, L.; Lin, S.; Rong, H.; Zheng, S.; Jin, S.; Wang, R.; Li, Y. Structural basis for iloprost as a dual peroxisome proliferator-activated receptor alpha/delta agonist. J. Biol. Chem. 2011, 286, 31473-31479. [CrossRef]

39. Otwinowski, Z.; Minor, W. Processing of X-ray diffraction data collected in oscillation mode. Macromol. Crystallogr. Part A 1997, 276, 307-326.

40. Vagin, A.; Teplyakov, A. MOLREP: An automated program for molecular replacement. J. Appl. Crystallogr. 1997, 30, 1022-1025. [CrossRef]

41. Emsley, P.; Lohkamp, B.; Scott, W.G.; Cowtan, K. Features and development of Coot. Acta Crystallogr. Sect. D-Biol. Crystallogr. 2010, 66, 486-501. [CrossRef] [PubMed]

42. Murshudov, G.N.; Skubak, P.; Lebedev, A.A.; Pannu, N.S.; Steiner, R.A.; Nicholls, R.A.; Winn, M.D.; Long, F.; Vagin, A.A. REFMAC5 for the refinement of macromolecular crystal structures. Acta Cryst. D Biol. Crystallogr. 2011, 67, 355-367. [CrossRef] [PubMed] 
43. Delano, W.L. The PyMOL Molecular Graphics System 2002. Available online: http://pymol.sourceforge.net/ overview/sld001.htm (accessed on 2 December 2019).

44. Nakano, S.; Yasukawa, K.; Tokiwa, T.; Ishikawa, T.; Ishitsubo, E.; Matsuo, N.; Ito, S.; Tokiwa, H.; Asano, Y. Origin of Stereoselectivity and Substrate/Ligand Recognition in an FAD-Dependent R-Selective Amine Oxidase. J. Phys. Chem. B 2016, 120, 10736-10743. [CrossRef] [PubMed]

45. Motoyama, T.; Nakano, S.; Yamamoto, Y.; Tokiwa, H.; Asano, Y.; Ito, S. Product Release Mechanism Associated with Structural Changes in Monomeric 1-Threonine 3-Dehydrogenase. Biochemistry 2017, 56, 5758-5770. [CrossRef] [PubMed]

46. Arulmozhiraja, S.; Matsuo, N.; Ishitsubo, E.; Okazaki, S.; Shimano, H.; Tokiwa, H. Comparative Binding Analysis of Dipeptidyl Peptidase IV (DPP-4) with Antidiabetic Drugs-An Ab Initio Fragment Molecular Orbital Study. PLoS ONE 2016, 11, e0166275. [CrossRef]

47. Molecular Operating Environment 2013 (MOE, Montreal, QC, Canada). Available online: https://www. chemcomp.com/Products.htm (accessed on 20 December 2019).

48. Ohtake, K.; Yamaguchi, A.; Mukai, T.; Kashimura, H.; Hirano, N.; Haruki, M.; Kohashi, S.; Yamagishi, K.; Murayama, K.; Tomabechi, Y.; et al. Protein stabilization utilizing a redefined codon. Sci. Rep. 2015, 5, 9762. [CrossRef]

49. Ishikawa, T.; Kuwata, K. Fragment molecular orbital calculation using the RI-MP2 method. Chem. Phys. Lett. 2009, 474, 195-198. [CrossRef]

50. Valiron, P.; Mayer, I. Hierarchy of counterpoise corrections for N-body clusters: Generalization of the Boys-Bernardi scheme. Chem. Phys. Lett. 1997, 275, 46-55. [CrossRef]

51. Kamiya, M.; Hirata, S.; Valiev, M. Fast electron correlation methods for molecular clusters without basis set superposition errors. J. Chem. Phys. 2008, 128, 074103. [CrossRef]

52. Tokiwa, T.; Nakano, S.; Yamamoto, Y.; Ishikawa, T.; Ito, S.; Sladek, V.; Fukuzawa, K.; Mochizuki, Y.; Tokiwa, H.; Misaizu, F.; et al. Development of an Analysis Toolkit. AnalysisFMO, to Visualize Interaction Energies Generated by Fragment Molecular Orbital Calculations. J. Chem. Inf. Model. 2019, 59, 25-30. [CrossRef]

(C) 2020 by the authors. Licensee MDPI, Basel, Switzerland. This article is an open access article distributed under the terms and conditions of the Creative Commons Attribution (CC BY) license (http://creativecommons.org/licenses/by/4.0/). 